\title{
Atrial natriuretic peptide in acute hypoxia-exposed healthy subjects and in hypoxaemic patients
}

\author{
F. Lordick*, R.W. Hauck*, R. Senekowitsch**, H.P. Emslander*
}

Atrial natriuretic peptide in acute hypoxia-exposed healthy subjects and in hypoxaemic patients. F. Lordick, R.W. Hauck, R. Senekowitsch, H.P. Emslander. (CERS Journals Ltd 1995.

ABSTRACT: Plasma atrial natriuretic peptide (ANP) is an endogenous vasodilator, which might counteract pulmonary hypertension due to hypoxaemia. The aim of this study was to investigate the influence of acute and chronic hypoxia on plasma (ANP) concentrations in humans.

Venous plasma ANP concentrations in six healthy subjects were measured before and after inhalation of hypoxic air $\left(11 \% O_{2}\right)$ at rest for $30 \mathrm{~min}$, and following 5 min of mild hypoxic exercise $(25 \mathrm{~W})$. Pulmonary arterial plasma ANP levels were determined in 31 right heart catheterized patients with lung disease.

In healthy subjects, mean arterial oxygen tension $\left(\mathrm{PaO}_{2}\right)$ was $6.8 \pm 1.9 \mathrm{kPa}$ after $30 \mathrm{~min}$ hypoxia at rest, and $4.7 \pm 0.9 \mathrm{kPa}$ after hypoxic exercise testing. Hypoxia at rest did not induce significant changes in ANP concentrations, whereas hypoxic exercise led to significantly increased ANP levels. Among hypoxaemic patients, significantly elevated plasma ANP levels were found only in the subgroup with increased mean pulmonary artery pressure, but not in the subgroup with normal pulmonary artery pressure.

In summary, severe acute hypoxia induced by hypoxic exercise, but not moderate acute hypoxia at rest, leads to increased ANP levels in healthy subjects. Chronic hypoxia alone does not lead to elevated plasma ANP levels; whereas, hypoxia combined with pulmonary hypertension causes increased plasma ANP concentrations in diseased subjects.

Eur Respir J., 1995, 8, 216-221.
*Medizinische Klinik, Pneumologie and **Nuklearmedizinische Klinik und Poliklinik, Technische Universität München, Klinikum rechts der Isar, München, Germany.

Correspondence: R.W. Hauck Medizinische Klinik

Technische Universität München

Klinikum rechts der Isar

Ismaninger Straße 22

D- 81675 München,

F.R.G.

Keywords: Acute hypoxia

atrial natriuretic factor

atrial natriuretic peptide

chronic hypoxia

lung disease

pulmonary hypertension

Received: January 211994

Accepted after revision October 151994
Atrial natriuretic peptide (ANP) is released into the circulation from right and left atrial myocytes. Atrial distension leads to an increased ANP release [1-5]. Previous animal studies have provided evidence for an augmented ANP release into the circulation during acute and chronic hypoxia [6-12]. Hypoxia-induced changes of the pulmonary haemodynamics may trigger this response $[10,11]$. The chemotropic effects of hypoxia leading to an enhanced ANP synthesis [11], as well as a reduction of the ANP plasma clearance [13], have also been discussed.

The ANP hormone leads to pulmonary vasodilatation, and might therefore, counteract pulmonary hypertension and right heart hypertrophy in hypoxic organisms [8, 14-18]. In patients with chronic obstructive lung disease (COLD), ANP infusion lowered the pulmonary artery pressure [19]. In contrast, the administration of an anti-ANP antibody aggravated pulmonary hypertension in hypoxia-adapted rats [15]. The blockade of endogenous ANP, however, failed to have a significant effect on pulmonary artery pressure during the first $6 \mathrm{~h}$ of hypoxic exposure, but led to an elevation of the pulmonary arterial pressure at later times. This suggests that endogenous ANP modulates the subacute, but not the acute phase of hypoxic pulmonary hypertension [20].
Most of the results from the above mentioned studies have been obtained from animal experiments. Little is known about ANP regulation during hypoxia in humans. Therefore, the aim of this study was to investigate whether hypoxia provides a relevant stimulus for an augmented ANP release in humans. Furthermore, we wanted to determine whether acute and chronic hypoxia should be regarded as two different entities in the regulation of ANP release.

Materials and Methods

Subjects

Six healthy volunteers (five males and one female) aged 20-24 yrs (mean 22 yrs), height 1.70-1.89 m (mean $1.81 \mathrm{~m}$ ), and weight $59-96 \mathrm{~kg}$ (mean $73 \mathrm{~kg}$ ) were investigated. Medical history, physical examination, 12-lead electrocardiogram (ECG) registration, and the results of an investigation of serum and blood parameters were within the normal range for each individual. Water and salt loading was examined by measuring the plasma sodium content. The plasma sodium content in all of 
the subjects was within the limits of normal range. None of the volunteers was taking any medication.

Thirty one patients ( 24 males, and 7 females) aged 49-78 yrs (mean 63 yrs), height 1.53-1.86 m (mean 1.70 $\mathrm{m}$ ), and weight 44-98 kg (mean $73 \mathrm{~kg}$ ) with various lung diseases, who had undergone right heart catheterization for diagnostic reasons, were admitted to the study. The following clinical diagnoses of lung diseases had been established: bronchial carcinoma $(n=24)$; bronchial carcinoma and $\operatorname{COLD}(n=2) ; \operatorname{COLD}(n=3)$; and lung resection due to bronchial carcinoma $(n=2)$. None of the subjects was undergoing corticosteroid therapy, and in all patients drug therapy (beta-adrenergic drugs, xanthine derivatives, calcium channel-blockers or nitrates) was stopped $36 \mathrm{~h}$ prior to the investigation.

Informed written consent was obtained from all of the healthy subjects and patients.

\section{Design of the study}

The study consisted of two protocols. Protocol 1 was designed to investigate the effects of acute normobaric hypoxia on plasma ANP release in six healthy volunteers. Protocol 2 was designed to assess the effect of chronic hypoxia on plasma ANP levels in 31 patients with various lung diseases.

Acute hypoxia was defined as an inducible hypoxaemia according to a sudden hypoxic stimulus of short duration. Hypoxaemia not fulfilling this condition was defined as chronic hypoxia.

Protocol 1. Examination of the volunteers started at 10.00 a.m. Subjects were placed in a supine position. Physiological variables monitored during the examination were: heart rate, systolic, diastolic and mean systemic arterial pressure, arterial blood gases including the arterial oxygen partial pressure $\left(\mathrm{PaO}_{2}\right)$, and the concentration of ANP in peripheral venous plasma (ANPven). The measurements were made at precisely defined time intervals, as follows: $\mathrm{C}=$ control measurements after $10 \mathrm{~min}$ in a supine position, breathing room air; $\mathrm{H}_{1}, \mathrm{H}_{2}$ and $\mathrm{H}_{3}$ i.e. after 10, 20, and $30 \mathrm{~min}$, respectively, of breathing a hypoxic gas with an inspiratory oxygen fraction $\left(\mathrm{FIO}_{2}\right)$ of $0.11 ; \mathrm{HE}=$ measurements after 5 min exercise with a bicycle ergometer (workload of $25 \mathrm{~W}$ ) in a supine position breathing a hypoxic gas $\left(\mathrm{FIO}_{2}=0.11\right) ; \mathrm{R} 1$ and $\mathrm{R} 2=$ measurements after 15 and 30 min recovery, respectively, breathing room air. The entire experiment lasted $75 \mathrm{~min}$.

Protocol 2. Examination of the patients also started at 10.00 a.m. As all the patients chosen for the study underwent right heart catheterization for diagnostic purposes, The following additional measurements were also performed: pulmonary arterial blood gases, mean right atrial pressure, mean pulmonary arterial pressure $(\overline{\mathrm{P}} \mathrm{pa})$, pulmonary capillary wedge pressure (PCWP), and cardiac output. Concentrations of ANP within the pulmonary artery plasma (ANPpa) were determined from routinely obtained blood samples. For the evaluation of the measurements, the patients were subdivided into four groups, according to their $\mathrm{PaO}_{2}$ and $\overline{\mathrm{P}}$ pa values at rest (fig. 1a and b): Group 1. Patients with arterial hypoxaemia and normal pulmonary artery pressure $(n=7)$; Group 2. Patients with arterial hypoxaemia and elevated pulmonary artery pressure $(n=5)$; Group 3 . Patients with arterial normoxaemia and elevated pulmonary artery pressure $(n=7)$; and Group 4. Patients with arterial normoxaemia and normal pulmonary artery pressure $(\mathrm{n}=12)$.

Arterial hypoxaemia and pulmonary hypertension were defined as follows. Arterial hypoxaemia: $\mathrm{PaO}_{2}(\mathrm{kPa})$ $<(86$ - age $\times 0.4) \times 0.1333+10 \%$. Pulmonary hypertension: $\mathrm{Ppa} \geq 2.7 \mathrm{kPa}(\geq 20 \mathrm{mmHg})$. There were no significant intergroup differences in the patients' age, height and weight.

\section{Methods}

Heart rate was measured via a continuously recorded ECG lead. Systemic arterial pressure was monitored by an indwelling 20-gauge polyethylene arterial cannula, within the left brachial artery, connected to a pressure transducer (PPG Hellige GmbH, Freiburg, FRG). All arterial blood samples were taken from the cannula within the left brachial artery. All peripheral venous blood samples were taken from an indwelling polyethylene cannula located in a left forearm vein; whilst all pulmonary artery blood samples were obtained from the proximal pulmonary artery lumen of a Swan Ganz catheter. Immediately after the blood samples were drawn, blood gas analysis was performed using a blood gas analyser (Ciba Corning Diagnostics GmbH, Fernwald, FRG) with automatic adjustment to atmospheric humidity and pressure.

Acute normobaric hypoxia was evoked in the healthy volunteers through inhalation of a gas mixture of $11 \%$ $\mathrm{O}_{2}$ and $89 \% \mathrm{~N}_{2}\left(\mathrm{FIO}_{2}=0.11\right)$, which was provided from a steel gas cylinder (Linde AG, Munich, FRG). The gas mixture was introduced by means of a mouthpiece connected to the gas cylinder via a plastic tube and a "demand valve" (Dräger Werke, Lübeck, FRG). A Y-valve excluded the possibility that expired air could be reinhaled.

Physical exercise was carried out in a supine position with a cycle ergometer (Erich Jaeger, Würzburg, FRG).

To facilitate right heart catheterization, a balloondirected and thermistor-tipped triple-lumen Swan Ganz catheter 7F (Spectramed, Düsseldorf, FRG) was positioned within the pulmonary artery via the left basilic vein or left internal jugular vein, using Seldinger's technique. The catheter was coupled to a pressure transducer (PPG Hellige GmbH, Freiburg, FRG), using the midaxillary line as zero reference level. Measurements of right atrial pressure (PRA), Ppa, and PCWP were performed by calculating the mean values of three respiratory cycles. The pressures and an ECG lead were recorded by a four-channel monitor and a four-channel writer (PPG Hellige GmbH, Freiburg, FRG). Cardiac output was determined by means of the thermodilution method using a Cardiac Output Computer ${ }^{\circledR}$ (Edwards Laboratories, Santa Ana, California, USA). Pulmonary 

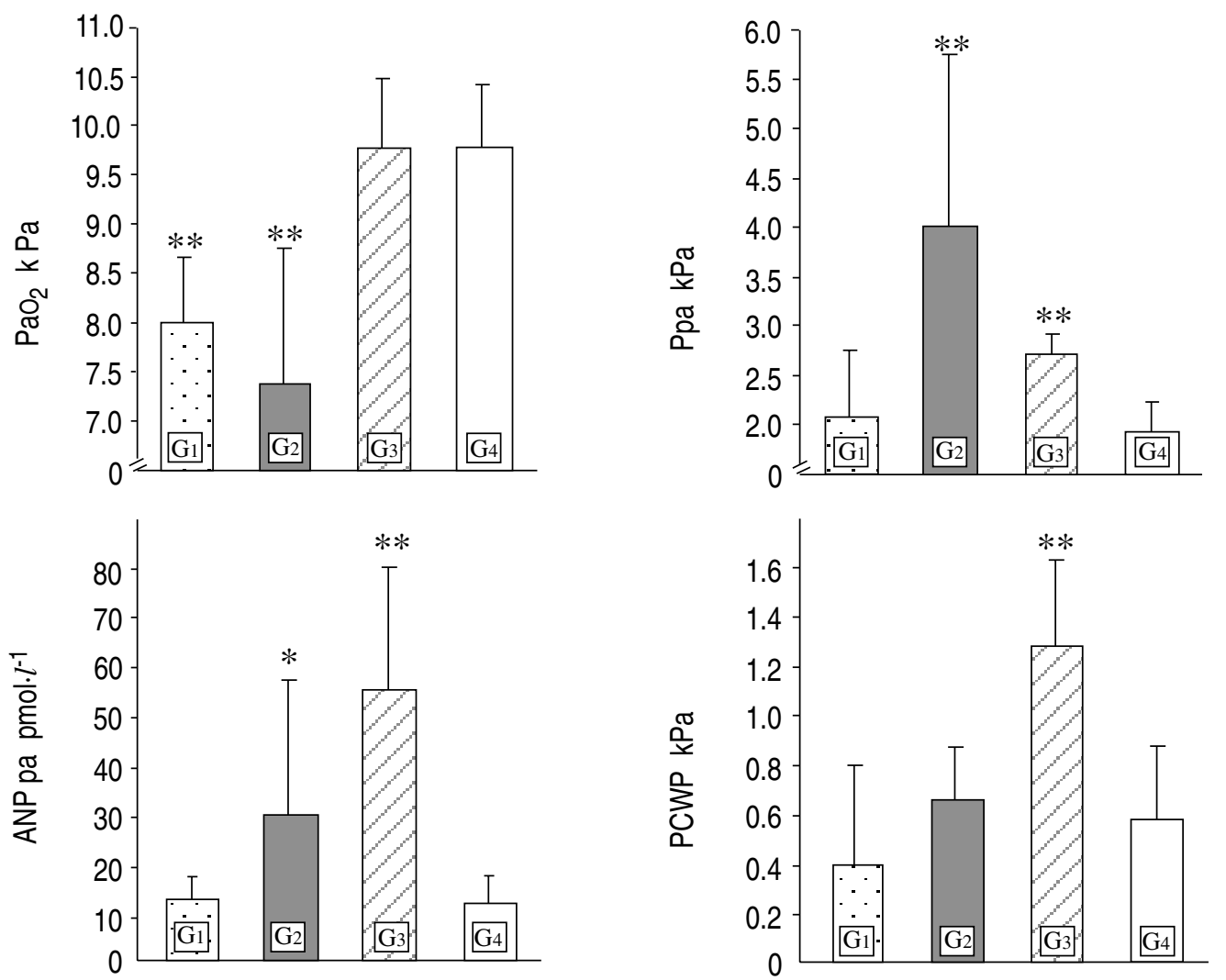

Fig. 1. - a) Arterial oxygen partial pressure $\left(\mathrm{PaO}_{2}\right)$; b) mean pulmonary arterial pressure (Ppa); c) concentrations of ANP in the pulmonary artery plasma (ANPpa); and d) pulmonary capillary wedge pressure (PCWP) were determined during right heart catheterization of 31 patients with various lung diseases. The patients were subdivided into four groups according to their $\mathrm{PaO}_{2}$ and Ppa values at rest (see a and b): $\mathrm{Gl}=\mathrm{hypox}-$

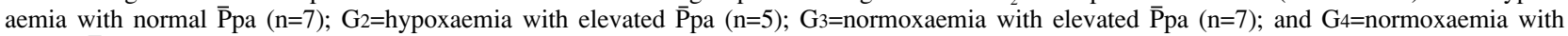
normal $\bar{P}$ pa $(n=12)$. c) $G_{2}$ and $G_{3}$ were significantly higher than $G_{1}$ and $G_{4}$, indicating that elevated plasma ANP levels in hypoxaemic patients only occur in the subgroup with pulmonary hypertension. d) G3 was significantly higher than $\mathrm{G} 1, \mathrm{G} 2$ and G4. Values represent mean \pm SD. $*: \mathrm{p}<0.05$ and $* *: \mathrm{p}<0.01$.

vascular resistance was calculated from a standard formula [21]. Blood for determination of plasma ANP levels was drawn into tubes coated with ethylene-diamine tetra-acetic acid (EDTA) and centrifuged for $10 \mathrm{~min}$ at $2,000 \times \mathrm{g}$ at $4^{\circ} \mathrm{C}$. Plasma was stored at $-70^{\circ} \mathrm{C}$ for a maximum of one month. Human ANP plasma concentrations were measured with the help of the commercially available radio-immunoassay ANP-RIAcid hANP (99126)® (Henning GmbH, Berlin, FRG) as described previously [21].

\section{Statistical analysis}

Data are expressed as mean values \pm standard deviations (SD). The effects of acute normobaric hypoxia, hypoxic exercise and recovery on plasma ANP levels and haemodynamic and blood gas values in healthy volunteers were assessed on paired data using the Wilcoxon signed-rank test. Analysis among and between more than two groups with different patient numbers was performed using the Kruskal-Wallis-test. For analysis between two groups, the Mann-Whitney test was used. The coefficients of correlation between plasma ANP levels and various blood gas and haemodynamic data were determined by linear regression analysis. Probabilities of less than 0.05 were considered as significant.

\section{Results}

\section{Protocol 1}

Concentrations of ANP within the venous plasma (ANPven) and $\mathrm{PaO}_{2}$ in six healthy study subjects are listed in table 1. After acute normobaric hypoxia, mean ANPven increased. However, this change was not statistically significant. In contrast, mean $\mathrm{PaO}_{2}$ decreased significantly $(\mathrm{p}<0.05)$ during this time period. After 5 min of exercise under hypoxic conditions (HE), ANPven increased in each individual. The plasma concentration was $31 \%$ higher than that in control conditions. This increase was statistically significant $(\mathrm{p}<0.05)$. The $\mathrm{PaO}_{2}$ further decreased and stood at a level which was significantly lower $(\mathrm{p}<0.05)$ than that after $30 \mathrm{~min}$ hypoxia at rest $\left(\mathrm{H}_{3}\right)$. Following recovery $(\mathrm{R} 1$ and $\mathrm{R} 2)$ both ANPven and $\mathrm{PaO}_{2}$ returned to baseline levels.

Regression analysis did not reveal any significant positive or negative coefficient of correlation between ANPven and arterial blood gas measurements. 
Table 1. - Concentrations of venous plasma ANP and $\mathrm{PaO}_{2}$ in six healthy study subjects exposed to normobaric hypoxia

\begin{tabular}{|c|c|c|c|c|c|c|c|}
\hline $\begin{array}{l}\text { Subject } \\
\text { No. }\end{array}$ & $\mathrm{C}$ & $\mathrm{H}_{1}$ & $\mathrm{H}_{2}$ & $\mathrm{H}_{3}$ & $\mathrm{HE}$ & $\mathrm{R} 1$ & $\mathrm{R} 2$ \\
\hline 1 & 3.5 & 3.5 & 3.5 & 3.5 & 4.5 & 3.0 & 3.0 \\
\hline 2 & 6.0 & 7.5 & 7.0 & 7.0 & 7.0 & 7.0 & 5.5 \\
\hline 3 & 4.0 & 4.5 & 5.0 & 5.0 & 6.5 & 5.5 & 5.5 \\
\hline 4 & 6.0 & 4.5 & 5.0 & 6.0 & 7.0 & 4.0 & 5.5 \\
\hline 5 & 10.0 & 9.0 & 11.0 & 11.0 & 13.5 & 11.0 & 10.5 \\
\hline 6 & 10.5 & 11.5 & 12.5 & 9.5 & 11.0 & 13.5 & 11.0 \\
\hline Mean & 6.5 & 7.0 & 7.5 & 7.0 & $8.5^{*}$ & 7.5 & 7.0 \\
\hline SD & 3.0 & 3.0 & 3.5 & 3.0 & 3.5 & 4.0 & 3.0 \\
\hline
\end{tabular}

\begin{tabular}{|c|c|c|c|c|c|c|c|}
\hline $\begin{array}{l}\text { Subject } \\
\text { No. }\end{array}$ & $\mathrm{C}$ & $\mathrm{H}_{1}$ & $\mathrm{H} 2$ & $\mathrm{H} 3$ & $\mathrm{HE}$ & $\mathrm{R} 1$ & $\mathrm{R} 2$ \\
\hline 1 & 11.7 & 4.9 & 4.8 & 4.8 & 3.7 & 12.3 & 12.9 \\
\hline 2 & 12.7 & 8.9 & 8.9 & 8.9 & 5.3 & 12.0 & 12.5 \\
\hline 3 & 12.0 & 8.9 & 9.6 & 8.0 & 6.0 & 10.9 & 12.9 \\
\hline 4 & 12.3 & 5.6 & 4.7 & 4.5 & 4.5 & 14.3 & 13.1 \\
\hline 5 & 12.7 & 6.1 & 6.5 & 6.8 & 4.0 & 10.2 & 12.1 \\
\hline 6 & 11.7 & 7.6 & 7.6 & 8.0 & 4.1 & 11.1 & 11.5 \\
\hline Mean & 12.3 & $7.1^{*}$ & $7.1^{*}$ & $6.8 *$ & $4.7 *$ & 11.9 & 12.5 \\
\hline SD & 0.5 & 1.7 & 2.1 & 1.9 & 0.9 & 1.4 & 0.7 \\
\hline
\end{tabular}

$\mathrm{C}$ : control (room air condition); $\mathrm{H}_{1}, \mathrm{H}_{2}, \mathrm{H} 3$ : 10, 20 and $30 \mathrm{~min}$ of normobaric hypoxia $\left(\mathrm{FIO}_{2}=0.11\right)$; HE: exercise testing under hypoxic conditions. $\left(\mathrm{FIO}_{2}=0.11\right.$; workload $\left.25 \mathrm{~W}\right) ; \mathrm{R} 1, \mathrm{R} 2$ : 15 and 30 min recovery (room air conditions); ANP: atrial natriuretic peptide; $\mathrm{PaO}_{2}$ : arterial oxygen partial pressure. FIO ${ }_{2}$ : inspiratory oxygen fraction. $*$ : $\mathrm{p}<0.05$ when compared with the values at control conditions $(\mathrm{C})$.

\section{Protocol 2}

The summary of the results in four groups (Groups $1-4)$ of 31 patients is illustrated in figure 1 . Note that in the hypoxaemic patients (Groups 1 and 2), significantly increased concentrations of pulmonary arterial plasma ANP (ANPpa) were found only in the subgroup with elevated $\bar{P}$ pa (fig. 1c).

Interestingly, the pattern of mean ANPpa measurements in the four groups was similar to the pattern of the pulmonary capillary wedge pressures (PCWP) (compare fig. 1c and d). ANPpa was significantly correlated with PCWP $(r=0.673 ; \mathrm{p}<0.01)$. However, regression analysis did not reveal a significant coefficient of correlation between ANPpa and any of the blood gas parameters measured in the systemic and pulmonary artery blood samples.

\section{Discussion}

In this study, we investigated whether hypoxia provides a stimulus for an augmented ANP release in humans. In healthy subjects, severe acute hypoxia caused an increase in plasma ANP levels. In diseased subjects with chronic hypoxia, elevated plasma ANP concentrations were detected only in the subgroup with pulmonary hypertension.

The results obtained in this study differed from the observations made in animal experiments, where inhalation of a hypoxic gas mixture had led to a significant increase of plasma ANP concentrations [7-10]. Although the healthy subjects at rest were exposed to a hypoxic gas mixture comparable to the gas mixtures used in the animal models, no significant ANP elevations in this condition were observed. However, the lack of statistical significance must be interpreted cautiously, as the number of the subjects was small in this study and minor effects of acute hypoxia on ANP release cannot be definitely excluded. The time of exposure to hypoxia at rest might have been too short to demonstrate a relevant stimulation of the ANP system. This possibility, however, appears unlikely, since in animal models under normobaric hypoxia plasma ANP levels peaked after 6-15 min [7, 8, 10], and in our study the hypoxic phase was extended to $30 \mathrm{~min}$.

As only a moderate arterial hypoxaemia was measured in the hypoxia-exposed subjects at rest, a further decrease of the $\mathrm{PaO}_{2}$ was evoked by mild hypoxic exercise. It should be remembered that the oxygen tension $\left(\mathrm{PO}_{2}\right)$ of the central venous blood, which was not monitored in this experiment, is even lower than the systemic arterial $\mathrm{PO}_{2}$ measured and may have been in the range of a perfusate used to evoke a chemotropically-induced release of ANP from isolated animal hearts [6]. Under these conditions, a significant increase in the venous plasma ANP concentration occurred. This suggests that severe acute hypoxia is a relevant stimulus for the ANP system in healthy humans.

Might the significant increase in plasma ANP levels be due to the physical exercise rather than to hypoxia? Although it has been shown that exercise is a potent 
trigger for an augmented ANP release in humans [22, 23], the intensity and duration of exercise used in our study were minimal. Therefore, we think that under our experimental conditions the severe acute hypoxia caused the increase in plasma ANP levels.

Previous studies in humans have yielded inconsistent results with respect to the influence of acute normobaric hypoxia on the plasma concentrations of ANP. LAWRENCE et al. [24] found a significant increase of the plasma ANP concentration within $30 \mathrm{~min}$ in healthy subjects breathing a gas mixture with $12 \%$ oxygen. Their study conditions, however, were different from the present study regarding dietary restrictions, premedication, and posture of the subjects during examination. MORICE et al. [25] did not observe any significant change in plasma ANP concentrations in subjects who had been exposed to normobaric hypoxia for $20 \mathrm{~min}$. This result was confirmed by Rock et al. [23], who exposed healthy subjects to hypobaric hypoxia for one hour. Similar results were also obtained by SchmidT et al. [22], who found that there was no significant effect of normobaric hypoxia on ANP concentrations in venous plasma samples from healthy subjects breathing air with a $12 \%$ oxygen content for $90 \mathrm{~min}$. However, in the present study, we showed that a supplemental hypoxic stimulus evoked by minimal exercise during hypoxia, causing a severe degree of hypoxaemia, can induce an elevation of plasma ANP levels.

After demonstrating that severe acute hypoxia has a significant effect on the ANP release in healthy humans, we wondered whether chronic hypoxia in patients with lung diseases leads to an elevation of plasma ANP concentrations. Various studies on rodents have shown that with chronic exposure to hypoxia, plasma ANP concentrations increased significantly [3, 4, 11, 12]. As pulmonary hypertension has been repeatedly reported to be a stimulus for ANP release into the plasma fluid of humans [25-27], we focused on the effect of mean pulmonary arterial pressure on ANP release. Our results indicate that in hypoxaemic patients with pulmonary hypertension, significantly increased concentrations of ANP within the pulmonary arterial plasma occur. Pulmonary hypertension in these patients was of the precapillary type. In animal models, atrial stretch appears to be a predominant stimulus for an increase of ANP release [5]. Therefore, it is possible that in these patients distension of the right atrium has led to the increase of plasma ANP concentrations. This is consistent with the results of BURGHUBER et al. [27], who demonstrated a strong correlation between plasma ANP concentrations and mean right atrial pressure in patients with precapillary pulmonary hypertension. In the present study, correlation of plasma ANP concentration with mean right atrial pressure was poor. However, right ventricular overload, which occurs in chronic pulmonary hypertension, may cause right atrial enlargement in the absence of increased atrial pressures [26]. Moreover, mean right atrial pressure does not accurately reflect the dynamic stretch of the right atrial myocardium, which may be the main trigger for an ANP release in patients with pulmonary hypertension [25].
In hypoxic patients without pulmonary hypertension (G4), plasma ANP concentrations were not elevated. It must be pointed out that in these patients the plasma ANP concentrations were similar to those seen in the subgroup of normoxaemic patients without pulmonary hypertension $(\mathrm{G} 1)$. Therefore, we suggest that chronic hypoxaemia alone is not a significant stimulus for an elevation of plasma ANP concentrations. In contrast, hypoxaemia with concomitant pulmonary hypertension is associated with increased plasma ANP concentrations. Taking into account that ANP can lower an increase of pulmonary arterial pressure in animals and humans [14$16,19,28]$, this may be a regulatory mechanism to counteract the pulmonary haemodynamic response in chronically hypoxaemic patients.

Previous studies in humans and animals have shown that not only the right but also the left atrium releases ANP if an adequate stimulus occurs [3, 29]. In the present study, the highest plasma ANP levels were observed in the subgroup of normoxaemic patients with pulmonary hypertension and an increase in the capillary wedge pressure, reflecting an overload of the left atrium. The underlying impaired left ventricular function was probably due to concomitant cardiovascular disorders, which have been present in these patients: arterial hypertension in two cases, arterial hypertension and coronary heart disease in four, and mild aortic valve stenosis in one. It can be suggested that the highly elevated plasma ANP concentrations in these patients can be attributed to the distension of the left atrium, as well as to an overload of the right atrium due to passive pulmonary hypertension.

We conclude that in healthy subjects at rest, acute normobaric hypoxia fails to induce a significant release of ANP into the plasma fluid. In contrast, at a severe degree of hypoxaemia, evoked by mild physical exercise under hypoxic conditions, plasma ANP concentrations increase significantly. In patients with lung diseases leading to chronic hypoxia, normal plasma ANP concentrations can be measured, as long as pulmonary haemodynamics are not altered. Therefore, hypoxia, per se, appears to be no relevant stimulus for an increase in plasma ANP concentrations in man. On the other hand, hypoxia together with supplemental factors, such as physical exercise in healthy subjects or pulmonary hypertension in patients, leads to significant elevations in plasma ANP levels.

Further investigations are needed to elucidate whether a therapeutic benefit can be obtained from augmented plasma ANP concentrations, for example by the administration of a neutral endopeptidase enzyme inhibitor [28].

\section{References}

1. Ballermann BJ, Brenner BM. Biologically active atrial peptides. J Clin Invest 1985; 76: 2041-2048.

2. de Bold AJ. Atrial natriuretic factor: a hormone produced by the heart. Science 1985; 230: 767-770.

3. Jin H, Chen YF, Yang RH, McKenna TM, Jackson RM, Oparil S. Vasopressin lowers pulmonary artery pressure 
in hypoxic rats by releasing atrial natriuretic peptide. Am J Med Sci 1989; 298 (4): 227-236.

4. McKenzie JC, Tanaka I, Inagami T, Misono KS, Klein RM. Alterations in atrial and plasma atrial natriuretic factor (ANF) content during development of hypoxia-induced pulmonary hypertension in the rat. Proc Soc Exp Biol Med 1986; 181: 459-463.

5. Dietz JR. Release of natriuretic factor from rat heartlung preparation by atrial distension. Am J Physiol 1984; 247 (Regulatory Integrative Comp. Physiol. 16): R1093-R1096.

6. Baertschi AJ, Hausmaninger C, Walsh RS, Mentzer RM, Wyatt DA, Pence RA. Hypoxia-induced release of atrial natriuretic factor (ANF) from the isolated rat and rabbit heart. Biochem Biophys Res Commun 1986; 140(1): 427-433.

7. Baertschi AJ, Adams JM, Sullivan MP. Acute hypoxaemia stimulates atrial natriuretic factor secretion in vivo. Am J Physiol 1988; 255 (Heart. Circ. Res. 24): H295-H300.

8. Adnot S, Chabrier PE, Brun-Buisson C, Viossat I, Braquet P. Atrial natriuretic factor attenuates the pulmonary pressor response to hypoxia. J Appl Physiol 1988; 65(5): 1975-1983.

9. Vachiery JL, Lejeune P, Hallemans R, et al. Atrial natriuretic peptides in canine hypoxic pulmonary vasoconstriction. Cardiovasc Res 1990; 24: 352-357.

10. Baertschi AJ, Teague WG. Alveolar hypoxia is a powerful stimulus for ANF release in conscious lambs. Am J Physiol 1989; 256 (Heart. Circ. Physiol 25): H990-H998.

11. Raffestin B, Adnot S, Mercadier JJ, et al. Synthesis and secretion of atrial natriuretic factor during chronic hypoxia: a study in the conscious instrumented rat. Clin Sci 1990; 78: 597-603.

12. Winter RJD, Meleagros L, Pervez S, et al. Atrial natriuretic peptide levels in plasma and in cardiac tissues after chronic hypoxia in rats. Clin Sci 1989; 76: 95-101.

13. Arnal F, Klinger JR, Warburton RR, Ou LC, Hill NS. Downregulation of lung atrial natriuretic peptide (ANP) binding sites in chronically hypoxic rats. Am Rev Respir Dis 1993, 147 (Suppl. 4): 725.

14. Jin H, Yang RH, Chen YF, Jackson RM, Oparil S. Atrial natriuretic peptide attenuates the development of pulmonary hypertension in rats adapted to chronic hypoxia. J Clin Invest 1990; 85: 115-120.

15. Raffestin B, Levame M, Eddahibi S, et al. Pulmonary vasodilatory action of endogenous atrial natriuretic factor in rats with hypoxic pulmonary hypertension. Circ Res 1992; 70 (1): 184-192.

16. Zhao L, Winter RJD, Krausz T, Hughes JMB. Effects of continuous infusion of atrial natriuretic peptide on the pulmonary hypertension induced by chronic hypoxia in rats. Clin Sci 1991; 81: 379-385.

17. Stewart AG, Barer GR, Morice AH. Effects of atrial natriuretic peptide (ANP) during hypoxic and $\mathrm{PgF}-2_{\alpha}$ vasoconstriction at high and low alveolar pressures. Eur Respir J 1991; 4 (Suppl. 14): Abstract 575.

18. Stewart AG, Thompson JS, Rogers TK, Morice AH. Atrial natriuretic peptide-induced relaxation of preconstricted isolated rat perfused lungs: a comparison in control and hypoxia-adapted animals. Clin Sci 1991; 81: 201-208.

19. Adnot S, Andrivet P, Chabrier PE, et al. Atrial natriuretic peptide in chronic obstructive lung disease with pulmonary hypertension. J Clin Invest 1989; 83: 986-993.

20. Jin H, Yang RH, Chen YF, et al. Atrial natriuretic peptide in acute hypoxia-induced pulmonary hypertension in rats. J Appl Physiol 1991; 71 (3): 807-814.

21. Hartter E, Woloszczuk W, Stummvoll HK. Radioimmunoassay of atrial natriuretic peptides in human plasma. Clin Chem 1986; 32 (3): 441-445.

22. Schmidt W, Brabant G, Kröger C, Strauch S, Hilgendorf A. Atrial natriuretic peptide during and after maximal and submaximal exercise under normoxic and hypoxic conditions. Eur J Appl Physiol 1990; 61: 398-407.

23. Rock PB, Kraemer WJ, Fulco CS, et al. Effects of altitude acclimatization on fluid regulatory hormone response to submaximal exercise. J Appl Physiol 1993; 75 (3): 1208-1215.

24. Lawrence DL, Skatrud JB, Shenker Y. Effect of hypoxia on atrial natriuretic factor and aldosterone regulation in humans. Am J Physiol 1990; 258 (Endocrinol. Metab. 21): E243-E248.

25. Morice AH, Pepke-Zaba J, Brown MJ, Thomas PS, Higenbottam TW. Atrial natriuretic peptide in primary pulmonary hypertension. Eur Respir J 1990; 3: 910-913.

26. Adnot S, Chabrier PE, Andrivet P, et al. Atrial natriuretic peptide concentrations and pulmonary hemodynamics in patients with pulmonary artery hypertension. Am Rev Respir Dis 1987; 136: 951-956.

27. Burghuber OC, Hartter E, Punzengruber C, Weissel M, Woloszuk W. Human atrial natriuretic peptide secretion in precapillary pulmonary hypertension. Chest 1988; 92 (1): 31-37.

28. Thompson JS, Sheedy W, Morice AH. The effects of neutral endopeptidase inhibition on the development of hypoxic pulmonary hypertension in rats. Am Rev Respir Dis 1993; 147 (Suppl. 4): 725.

29. Raine AEG, Erne P, Bürgisser E, et al. Atrial natriuretic peptide and atrial pressure in patients with congestive heart failure. $N$ Engl J Med 1986; 315: 533-537. 